\title{
Actions de prévention, annonce des crues et protection des lieux habités
}

\author{
par M. Claude Truchot, \\ Directeur régional de l'environnement d'Ile-de-France
}

\section{I $\square$ CADRE NATIONAL DE LA PRÉVENTION DES RISQUES D'INONDATIONS}

Une politique de prévention des risques d'inondation et de gestion des zones inondables a été arrêtée par le gouvernement le 13 juillet 1993 .

Un programme d'action pour la prévention des risques naturels a été décidé par le gouvernement lors d'un comité interministériel du 24 janvier 1994.

\section{- 1.1 Politique de prévention des risques d'inondation}

Les objectifs de la politique de l'Etat en matière de gestion des zones inondables précisés par une circulaire interministérielle du 24 janvier 1994 (parue au J. O.) sont les suivants :

- interdire les implantations humaines dans les zones où la sécurité des personnes ne peut être garantie intégralement et les limiter dans les autres zones inondables,

- préserver les capacités d'écoulement et d'expansion des crues,

- sauvegarder l'équilibre des milieux dépendant des petites crues et la qualité des paysages des vallées.

Les principes à mettre en œuvre pour la prévention des inondations sont les suivants :

\section{ler principe :}

- à l'intérieur des zones inondables soumises aux aléas les plus forts interdire toute construction nouvelle et réduire le nombre de constructions exposées,

- dans les autres zones inondables prendre des dispositions pour réduire la vulnérabilité des constructions qui pourront être éventuellement autorisées et inciter les autorités locales et les particuliers à prendre des mesures adaptées pour les habitations existantes.

\section{2ème principe :}

- contrôler strictement l'extension de l'urbanisation dans les zones d'expansion des crues (secteurs non urbanisés ou peu urbanisés et peu aménagés),

- veiller fermement à ce que les constructions qui pourront être éventuellement autorisées soient compatibles avec les impératifs de la protection des personnes, de l'écoulement des eaux et des autres règlementations (protection des paysages, sauvegarde des milieux naturels).

3ème principe :

- éviter tout endiguement ou remblaiement nouveau qui ne serait pas justifié par la protection des lieux fortement urbanisés.

\subsection{Programme d'action pour la prévention des risques naturels}

Un programme d'action de 11 milliards de francs pour la prévention des risques naturels, financé globalement à $45 \%$ par l'Etat, précisé par une circulaire du Ministère de l'Environnement du 27 janvier 1994 (non parue au J.O.), comprend en particulier les mesures suivantes :

- simplifier la procédure d'élaboration des plans d'identification des risques en fusionnant les procédures, PER, PSS, Périmètre de risque R111-3 du C.U. en une nouvelle procédure appelée plan de prévention des risques (PPR) (loi relative au renforcement de la protection de l'environnement),

- accélérer le rythme d'élaboration des plans d'identification des risques (2000 communes prioritaires d'ici 5 ans),

- donner des instructions aux préfets pour la maîtrise de l'urbanisation dans les zones inondables (circulaire du 24 janvier 1994),

- inviter les préfets à faire usage du code de l'urbanisme pour contrôler les aménagements dans les zones fortement

Inhabited areas protection against floods includes three periods : - prevention with the application of flood risks prevention plans, - preventive information concerming phenomena evolution (starting from measurements and forecasting tools), - the building of protection works after thinking about their influence on the upper and down-stream waters. 
touchées par les récentes inondations (circulaire du Premier Ministre du 2 février 1994),

- adopter un décret qui permettra aux préfets d'imposer des mesures de sécurité aux campings (décret $\mathrm{n}^{\circ}$ 94-614 du 13 juillet 1994),

- améliorer le système d'annonce des inondations,

- engager un plan décennal de restauration et d'entretien des rivières d'un montant global de 10,2 milliards de francs, financé par l'Etat à hauteur de $40 \%$, qui comporte 3 volets : - la restauration des cours d'eau,

- la protection des lieux habités contre les crues,

- l'équilibre écologique des cours d'eau,

- dynamiser l'entretien des cours d'eau en liant la participation financière de l'Etat à la réalisation de plan simple de gestion.

\section{II $\square$ ACTIONS RÉGLEMENTAIRES DE PRÉVENTION DES RISQUES D'INONDA- TIONS EN RÉGION PARISIENNE}

\section{- 2.1. Les Plans de Prévention des Risques (P.P.R.) natu- rels prévisibles d'inondations}

Depuis le 11 octobre 1995, date de parution au J.O. du décret $\mathrm{n}^{\circ}$ 95-1089 du 5 octobre 1995 relatif aux plans de prévention des risques naturels prévisibles, les plans d'exposition aux risques naturels prévisibles (P.E.R.), plans de surfaces submersibles (P.S.S.) et périmètres de risques (P.R.) délimités en application de l'article R. 111-3 du code de l'urbanisme approuvés en région Ile-de-France valent P.P.R. et ceux en cours d'élaboration sont des projets de P.P.R.

Actuellement sur la Seine (figure 1), dans le département des Yvelines ce sont d'anciens P.S.S. et P.R. valant P.P.R. inondation qui sont approuvés ; il existe quelques P.E.R. valant P.P.R. inondation approuvés sur quelques communes du Val-d'Oise ; en Essonne des P.P.R. inondation sont en projet ; en Seine-et-Marne un P.P.R. inondation, sur le secteur de Montereau, est en projet. Dans nombre de communes comme dans le Val-de-Marne il existe des limites de zones inondables dans le cadre des servitudes des Plans d'Occupation des Sols.

Sur la Marne, d'anciens P.S.S. valant P.P.R. inondation sont approuvés dans la Seine-et-Marne.

Sur l'Oise, des P.P.R. inondation sont en projet dans le Val d'Oise.

En revanche, le Val-de-Marne, Paris, les Hauts-de-Seine et la Seine Saint-Denis ne sont couverts par aucun document réglementaire.

Il existe actuellement une hétérogénéité dans la prise en compte des risques d'inondations le long de la Seine, la Marne et l'Oise en région parisienne.

Les travaux d'élaboration des P.P.R. réalisés par les Directions Départementales de l'Equipement de la région parisienne sont aidées par le ministère de l'Environnement pour un montant d'environ $2 \mathrm{MF}$ par an.

La DIREN Ile-de-France et la DRE Ile-de-France animent actuellement un groupe de travail, avec les services du SNS et des DDE, chargé en particulier de définir un cadre de références techniques pour l'élaboration des P.P.R. inondation.

L'objectif fixé par le ministère de l'Environnement est la couverture par des P.P.R. des communes à risques avant l'an 2000.

\subsection{Les actions d'information préventive}

Conformément à l'article 21 de la loi $n^{\circ} 87-585$ du 22 juillet 1987 et au décret d'application $\mathrm{n}^{\circ}$ 90-918 du 11 octobre 1990 pris pour son application, les Préfets des départements de la région parisienne ont tous constitué une commission d'analyse des risques et d'information préventive (C.A.R.I.P.) chargée d'élaborer un dossier départemental des risques majeurs (D.D.R.M.) et des dossiers communaux synthétiques (D.C.S.).

Ces D.C.S. doivent permettre aux maires de réaliser les documents d'information communale sur les risques majeurs (D.I.C.R.I.M.) qui doivent être portés à la connaissance des populations concernées.

Les D.D.R.M. ont été réalisés dans les départements de Seine-et-Marne, de l'Essonne, des Hauts-de-Seine, de SeineSaint-Denis et du Val-de-Marne, et sont en projet dans les départements de Paris, des Yvelines et du Val-d'Oise. Très peu de D.C.S. ont été réalisés et notifiés aux maires concernés.

Les inondations dues aux crues de la Seine, de la Marne et de l'Oise en région parisienne ont été prises en compte dans tous les D.D.R.M.

\section{III - L'ANNONCE DES CRUES EN ILE-DE-FRANCE}

\subsection{La mission d'annonce des crues}

Face au phénomène naturel que constituent les crues et aux catastrophes qu'elles peuvent engendrer, l'Etat, sans en avoir l'obligation légale, a organisé sur certaines rivières, l'annonce des crues et la transmission des avis de crues. En application du code des communes, le maire, autorité de police administrative, reste toutefois responsable de la sécurité publique sur le territoire communal ; il est par conséquent chargé de prévenir ses administrés contre la montée des eaux. Il est l'interlocuteur privilégié des services locaux et nationaux des ministères concernés, qui doivent l'alerter, faire en sorte qu'il soit informé et l'aider dans ses tâches de secours. Le règlement départemental d'annonce des crues et l'action d'information que le Préfet mène pour le faire connaître, doivent mettre ce rôle en valeur et faire clairement apparaitre que cette organisation n'a d'autre but que d'en faciliter l'exercice.

Cette mission consiste dès lors pour les services d'annonce des crues, services de l'Etat, en la surveillance des cours d'eau, l'élaboration des méthodes permettant l'annonce des crues ainsi que la mise en œuvre de ces méthodes pendant la période de crues.

\section{- 3.2 Encadrement réglementaire}

Au plan national, elle est encadrée par deux arrêtés interministériels :

— arrêté interministériel du 27 février 1984 portant réorganisation de l'annonce des crues et de la transmission des avis de crues et ses annexes,

— arrêté interministériel du 27 février 1984 portant réorganisation des services d'annonce des crues et ses annexes, modifié par arrêté du 24 septembre 1986 et par arrêté du 19 mars 1993.

Les rivières concernées sont celles où l'Etat a décidé d'assumer ce service en raison des enjeux du point de vue de la sécurité publique (zones inondables, populations concernées, ...) et de la faisabilité (délais d'alerte, conditions financières, techniques et humaines de mise en œuvre). 


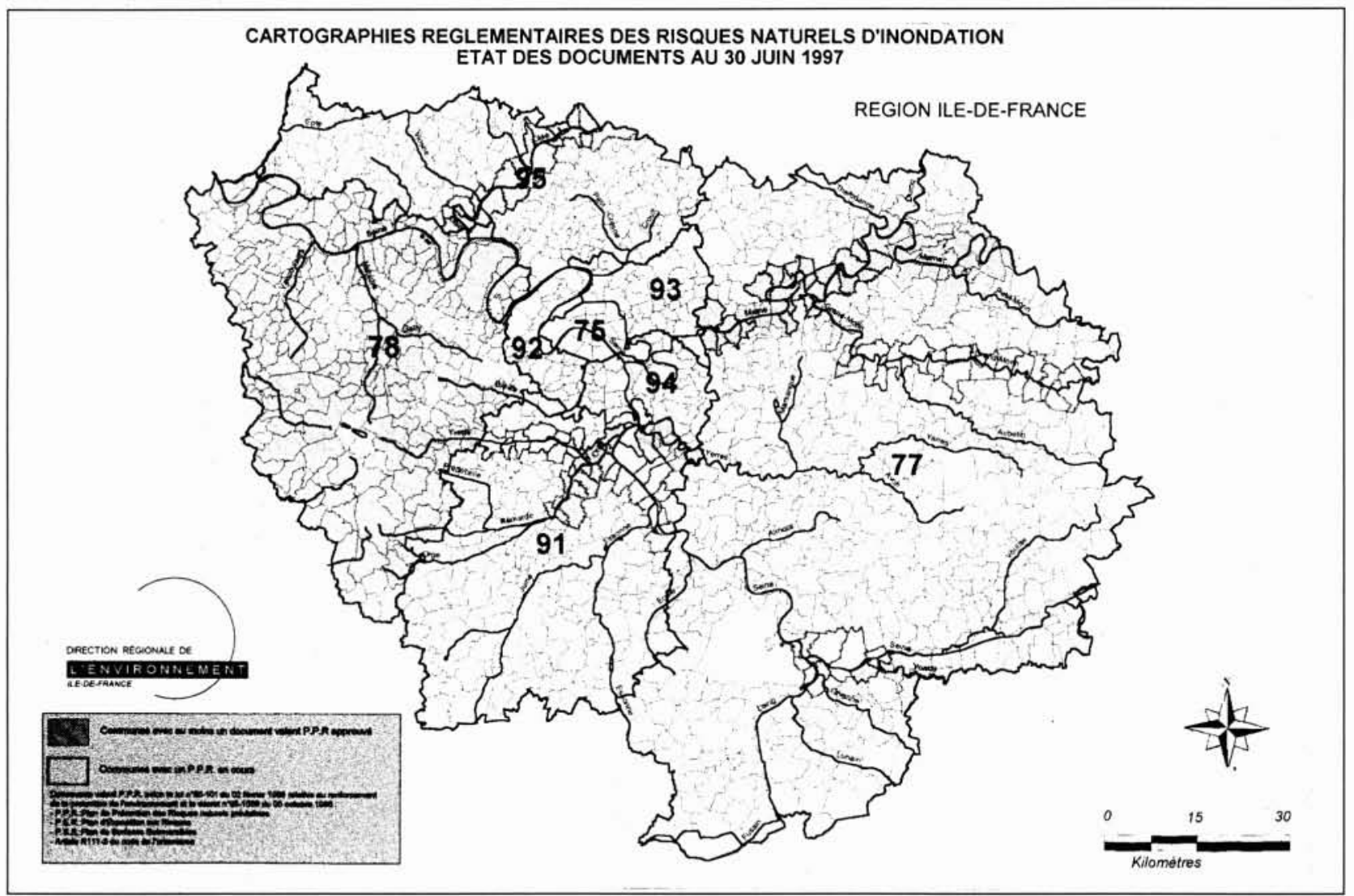

1. Cartographies réglementaires des risques naturels d'inondation - Etat des documents au 30 juin 1997

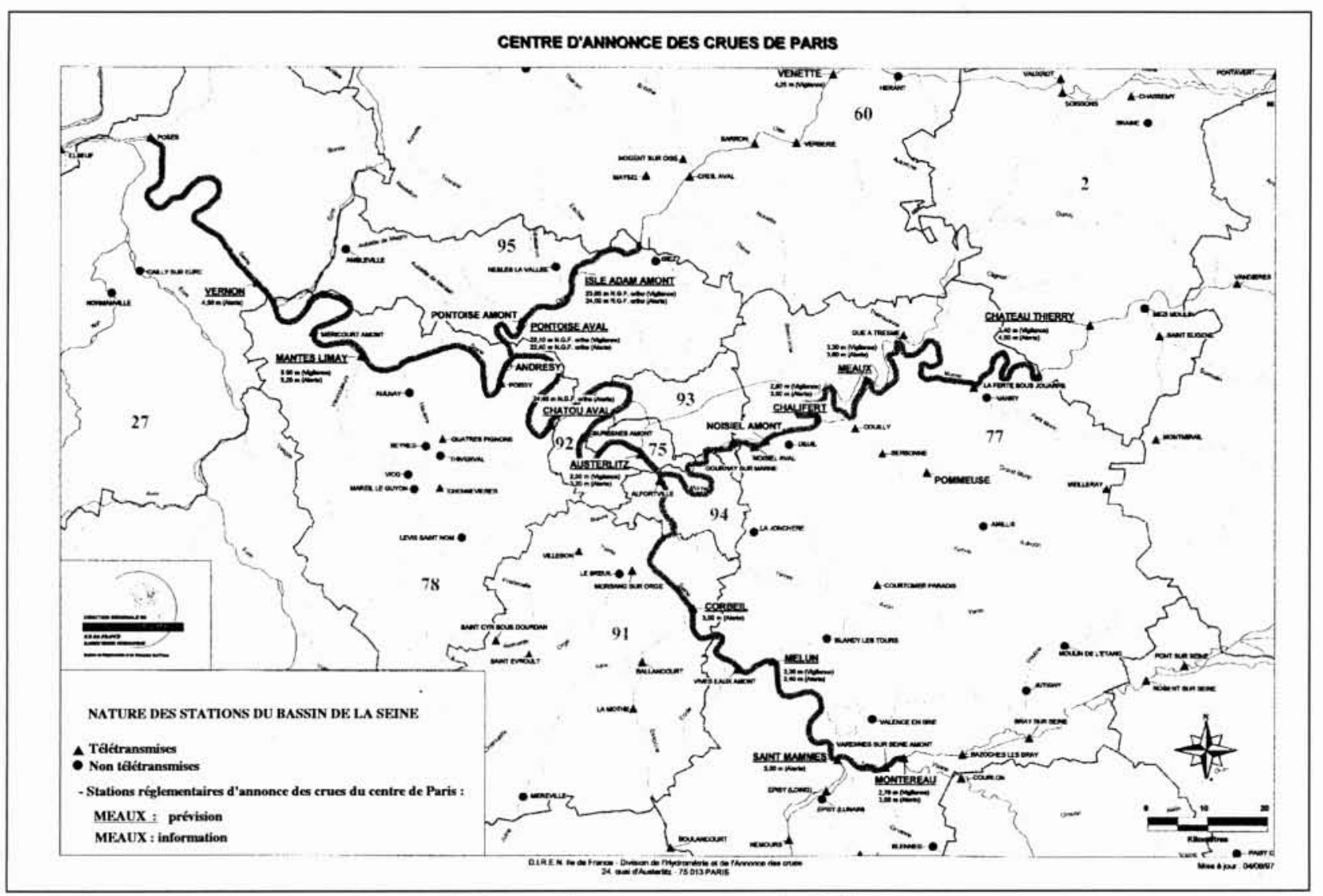

2. Centre d'annonce des crues de Paris. 
Au plan local, cette mission est réalisée dans le cadre de règlements départementaux d'annonce des crues approuvés par le Préfet qui prévoient les conditions de vigilance des centres d'annonce, les conditions de mise en alerte ainsi que les modalités d'information sur l'évolution de la crue.

\section{- 3.3 Principes d'organisation} 1984

Deux principes essentiels ont guidé la réorganisation de

Le premier principe est un principe d'unicité ; il consiste en la clarification des responsabilités entre les services concernés par la transmission des avis de crues, l'organisation de cette transmission devant en effet être la même sur tout le territoire national, la mission de chaque service étant nettement définie et chaque agent connaissant son rôle ainsi que celui de ses interlocuteurs.

Le deuxième principe est un principe de séparation entre les processus d'alerte et d'information.

Le processus d'alerte se compose de deux phases : une phase initiale qui consiste à recueillir et traiter des informations hydrométéorologiques en vue de la préparation des messages d'alerte et qui est de la compétence des services d'annonce des crues. Une seconde phase relative à la décision d'alerter les maires prise par le Préfet de chaque département, la transmission du message étant assurée sous son autorité par les personnels des services de protection civile, d'incendie et de secours, de gendarmerie et de police.

S'agissant du processus d'information ultérieure sur l'évolution de la crue, l'organisation donne aux maires un rôle actif dans le recueil des renseignements. Ils doivent en effet, après avoir été alertés, s'informer eux-mêmes sur son déroulement en appelant au moyen d'un numéro de téléphone qui leur est réservé, un serveur vocal dont le fonctionnement est assuré sous l'autorité du Préfet et alimenté par les services de protection civile à partir des informations apportées par les services d'annonce des crues.

\section{- 3.4 Cas de l'Ile-de-France}

La DIREN Ile-de-France gère un réseau de 133 stations télétransmises sur l'ensemble du bassin de la Seine (figure 2). Une bonne partie de ces stations sont nécessaires à l'annonce des crues. Les autres ont une vocation purement hydrométriques. Les données fournies par ces stations permettent aux 8 centres d'annonce des crues du bassin de la Seine (Corbigny, Montargis, Sens, Troyes, Paris, Chaumont, Reims et Compiègne) d'assurer leur mission.

La responsabilité de l'annonce des crues en Ile-de-France est partagée entre quatre services :

— La DDE de la Nièvre à Montargis (Loing),

- Le Service de la navigation de la Seine à Sens (Yonne),

- La DDE de l'Aube à Troyes (Seine à l'amont de la confluence avec l'Yonne),

- La DIREN à (Seine à l'aval de la confluence avec l'Yonne, Marne et Oise).

Dès que certaines cotes sont atteintes aux stations réglementaires, le service d'annonce des crues informe le(s) préfet(s) concerné(s) que le service s'est mis en état de vigilance ou lui propose de déclencher l'alerte. Il le(s) tient régulièrement informé(s) de l'évolution de la situation en complétant les informations des stations réglementaires par les cotes aux stations d'information.

La DIREN Ile-de-France assure l'annonce des crues pour
18 stations dont 16 sont télétransmises. Elles se répartissent de la manière suivantes :

Bassin de la Marne : Château Thierry (vigilance et alerte)

Meaux (vigilance et alerte), Pommeuse sur le Grand Morin (information), Chalifert (vigilance et alerte).

Bassin de l'Oise : Venette (vigilance), Isle Adam (vigilance et alerte), Pontoise amont (information), Pontoise aval (vigilance et alerte).

Bassin de la Seine : Montereau (vigilance et alerte), Saint Mammès (alerte), Melun (vigilance et alerte), Corbeil (alerte), Paris-Austerlitz (vigilance et alerte), Chatou (alerte), Andrésy (information), Mantes-Limay (vigilance et alerte), Vernon (alerte).

La DIREN Ile-de-France mène actuellement trois actions pour améliorer son action en matière d'annonce des crues :

- Réorganisation et modernisation du réseau de mesures. Il s'agit de rationaliser la gestion du réseau afin d'en faciliter la maintenance et d'en amélioration la fiabilité.

- Développement d'outils de prévision permettant d'amélioration la précision des prévisions.

- Mise en auvre durant l'hiver 1996-97 d'une démarche expérimentale associant le centre d'annonce des crues, les préfectures de Seine-et-Marne, de Seine-Saint-Denis et du Val d'Oise et trois communes volontaires (Condé-SainteLibiaire, Gournay-sur-Marne et Auvers-sur-Oise) et ayant pour objectif de permettre la valorisation par les communes des données de l'annonce des crues. Cette démarche permet aux communes de transformer les données des stations d'annonce des crues en données à une échelle installée au droit de la commune et de mieux délimiter les secteurs susceptibles d'être inondés.

\section{IV $\square$ TRAVAUX DE PROTECTION DES LIEUX HABITÉS EN RÉGION PARISIENNE}

Le contrat de plan signé le 20 juillet 1994 entre l'Etat et la Région Ile-de-France retient, en son article 7, le financement d'un programme de travaux de protection des lieux habités contre les inondations le long des rivières domaniales et non domaniales. Les études et travaux aidés sont arrêtés chaque année par concertation entre l'Etat et la Région Ile-de-France au vu des demandes des maîtres d'ouvrage. Le taux de subvention, pour l'Etat comme pour la Région, est fixé à $25 \%$ du montant hors taxes des études et des travaux. Le montant total de financement retenu pour les travaux de protection par le contrat de plan s'élèvent à 29,25 MF pour l'Etat comme pour la Région Ile-de-France.

Le ministère de l'Environnement a délégué à la Préfecture de Région, au titre des dotations 1994, 1995, 1996 et 1997 pour l'actuel contrat de plan, un montant total d'A.P. sur le chapitre 67-20 article 20 de 19700000 francs. 29 opérations nouvelles de protection ont été individualisées en C.A.R. Il s'agit pour l'essentiel de travaux réalisées par les collectivités territoriales (départements, syndicats intercommunaux et communes) de construction ou d'amélioration de digues et murettes anti-crue, de stations de pompage anti-crue, d'ouvrages de régulation hydrauliques et de bassins de retenue.

Ces travaux ne visent pas à protéger les lieux habités contre les crues centennales ou du type de la crue de 1910, mais contre des crues plus fréquentes et d'intensité moyenne. 